neonatal infection, it is mentioned in some studies that MSAF is a risk factor for neonatal infection. Knowledge about the types of pathogens is still limited and pathogens is curiosity.

Objective Determine pathogens contain in MSAF which lead to neonatal infection in newborn with MSAF.

Method Cohort study. Subjects newborns with MSAF delivered in RS. Dr. Kariadi from October 2009 - March 2010 with inclusion criteria. MSAF was determined by KAPPA test (0.74) and contain one of stool metabolite. Group II was babies with clear amniotic fluid. Examination of variables were taken on the first day. Statistical analysis used chi square, Mann whitney, and relative risk (CI 95\%).

Result Subjects were 70 babies. Group I: 35 baies and Group II: 35 . Babies with MSAF and viscous amniotic fluids have $10 \mathrm{x}$ higher risk to be infected ( $95 \% \mathrm{CI}=1.3-74.0 ; \mathrm{p}=0,003)$. Incidence of neonatal infection by Gram staining: Gram (+) has RR 1.4 (95\%CI=0.3- -6.8; $\mathrm{p}=0.6$ ) and incidence of both Gram (+) and Gram (-) has RR 2.4 (95\%CI=0.7-7.7; $\mathrm{p}=0.2$ ). RR of babies with MSAF containing E coli culture become sepsis was $3.8(95 \% \mathrm{CI}=0.8-17.0 ; \mathrm{p}=0,057)$ and non E coli culture was 2.4 (95\%CI $=0.4-13.1 ; \mathrm{p}=0.4$.

Conclusion E coli was the prominent pathogen in babies with MSAF but not a risk factor. MSAF is the risk factor for neonatal infection.

\section{INCIDENCE AND ORGANISAM PATTERN IN EARLY ONSET NEONATAL SEPSIS}

doi:10.1136/archdischild-2012-302724.1176

'LZ Hajnal Avramovic, 'TP Lazic Mitrovic, 'M Rascanin, ${ }^{1} \mathrm{~J}$ Korac, ${ }^{2} \mathrm{~A}$ Curkovic. 'Neonatal Department; ${ }^{2}$ Maternity Department, GAK 'Narodni Front', Belgrade, Serbia

Background and Aim Early onset neonatal sepsis (EONS) occurs within the first 3 to 7 days of life. The incidence of EONS vary from 1 to 4.6 cases per 1000 live newborns. The distributions of organisms in EONS helps to use appropriate antibiotics prophylaxis during labour and neonates with suspected sepsis. The aim of our study was to compare the incidence and the organisms distribution for EONS during 2009, 2010 and 2011 for infants admitted to NICU in our Neonatal Department.

Methods Data were retrieved from newborns with positive bacterial blood and/or cerebral spinal fluid in the first $72 \mathrm{~h}$ after birth. We compared incidence rate and causative organisms.

Results A total of 198 newborns with suspected sepsis, 125 had positive cultures over the time of three years period. The EONS incidence was 8.1 (54 per 6659 neonates) in 2009, 5.7 (40 per 6994 neonates) in 2010. and 4.5 (31 per 6883 neonates) in 2011. B Streptococcus were the most common organism (3.4/1000) in the term infants. Staphylococcus coagulase-negative was second with rate 2.8/1000. Escherichia coli (3.8/1000) and Staphylococcus coagulase-negative (3.5/1000) were the most common in preterm infants. There were no significant changes in organism pattern in EONS during study period.

Conclusion The rate of EONS among neonates in NICU in study period was not significantly changed and we did not find significant change in bacterial organisms. So, we suggest further prevention of EONS focused on prevention of vertical transmission and intrapartum antibiotics prophylaxis.

\section{IMPACT OF 4\% CHLORHEXIDINE CORD CLEANSING OF UMBILICAL CORD ON BACTERIAL GROWTH OF NEWBORNS IN PEMBA, TANZANIA}

doi:10.1136/archdischild-2012-302724.1177

${ }^{1} S$ Sazawal, ${ }^{1}$ U Dhingra, ${ }^{2} \mathrm{~S}$ Madhesiya, ${ }^{3} \mathrm{~A}$ Dutta, ${ }^{3} \mathrm{SM}$ Ali, ${ }^{3} \mathrm{~S}$ Ame, ${ }^{3} \mathrm{~S}$ Deb, ${ }^{1} \mathrm{R}$ Black. IInternational Health, Johns Hopkins Bloomberg School of Public Health, Baltimore, MD, USA; ${ }^{2}$ Center for Public Health Kinetics, New Delhi, India; ${ }^{3}$ Public Health Laboratory-IdC, Chake Chake Pemba, Zanzibar, Tanzania
Introduction Studies in Nepal, Pakistan, and Bangladesh have shown using 4\% CHX solution for umbilical cord cleansing reduces neonatal mortality and omphalitis. Data evaluating the effect of $4 \%$ Chlorhexidine umbilical cord cleansing from the Sub-Saharan region is lacking. Considering this need we are undertaking a double blind, controlled study in Eastern Africa. Before starting the trial, in this pilot we tested the impact of $4 \%$ Chlorhexidine and control solution specially prepared for the trial on colonization and colony count.

Methods Total 512 newborns in both the hospital and community were enrolled in the study. Newborns were randomly assigned the Chlorhexidine, placebo or dry cord care group. Umbilical swabs were collected at baseline (before the application of intervention), 2 hour and 48 hour after application of the assigned intervention. Presence of growth, identification to gram positive/ negative groups and semi-quantitative colony count was estimated for all samples.

Results The positivity was high baseline swabs 30\% (154 of 512 samples). In 2 hour post intervention group Chlorhexidine significantly reduced the growth of pathogens compared to placebo (OR $0.15, \mathrm{p}<0.01$ ) and dry cord [OR 0.07, $\mathrm{p}=0.00$ ]. In 48-hour swabs reduction in growth and density of organisms was observed in Chlorhexidine group (OR 0.11, $\mathrm{p}<0.01$ ). There was no difference between the control solution and dry cord group (OR 0.97, $\mathrm{p}=0.92$ ). Conclusions Chlorhexidine preparation was effective in reducing the growth and density of pathogens over the umbilical cord. The control preparation did not increase colonization but was similar to dry cord care group.

\section{EVALUATING OPTIMAL QUANTITY OF CHLORHEXIDINE SOLUTION NEEDED FOR APPLICATION TO UMBILICAL CORD OF NEONATES IN FIRST 10 DAYS OF LIFE}

doi:10.1136/archdischild-2012-302724.1178

${ }^{1} \mathrm{~S}$ Sazawal, ${ }^{2} \mathrm{~S}$ Nangia, ${ }^{3} \mathrm{~S}$ Deb, ${ }^{4} \mathrm{~S}$ Madhesiya, ${ }^{3} \mathrm{~A}$ Dutta, ${ }^{4} \mathrm{P}$ Dhingra, ${ }^{1} \mathrm{U}$ Dhingra, ${ }^{3} \mathrm{SM}$ Ali, ${ }^{3} \mathrm{~S}$ Ame, ${ }^{4} \mathrm{~S}$ Gupta, ${ }^{4} \mathrm{~A}$ Ahmed, 'R Black. ' Johns Hopkins University Bloomberg School of Public Health, Baltimore, MD, USA; '2Department of Pediatrics, Lady Hardinge Medical College \& Kalawati Saran Children's Hospital, New Delhi, India; ${ }^{3}$ Public Health Laboratory-IdC, Chake Chake Pemba, Zanzibar, Tanzania; ${ }^{4}$ Center for Public Health Kinetics, New Delhi, India

Background Efficacy studies of application of chlorhexidine on umbilical cord have suggested significant improvement in neonatal outcomes. An important question for new trials and programs however is what should be the quantity used. There are concerns about the increased risk of hypothermia resulting from spillage or over use of any cleansing liquid solution in newborn. In context of a randomized controlled trial evaluating impact of cord cleansing in Africa, on recommendation of DSMB we undertook a pilot study, which aimed to determine the optimal quantity of the intervention solution required for application on umbilical cord of newborn.

Methods Children were enrolled from both community and hospital in Pemba $(n=62)$ and only from Hospitals in Delhi $(n=50)$. Trained Hospital staff/MCH applied the intervention solution from a dropper bottle filled with $10 \mathrm{ml}$, on the umbilical cord of the baby generously such that it covered umbilical cord and periumbilical area. A study supervisor to maintain consistency supervised the process. After application the unused volume from each of the containers was measured to determine the actual usage.

Results The mean volume of usage did not differ between Pemba and Delhi $(4.58 \pm 0.8 \mathrm{ml}$ and $4.79 \pm 1.88 \mathrm{ml}$ respectively). The quantity of solution used ranged from $3 \mathrm{ml}$ to $7.5 \mathrm{ml}$ with a median of $4.5 \mathrm{ml}$.

Conclusions The optimal requirement for application was found to be $5 \mathrm{ml}$. However to be little conservative we recommend use 6 $\mathrm{ml}$ to adjust for any spillage and/or any abnormally long cord. 